International Journal of Pure and Applied Mathematics

Volume 86 No. 2 2013, 377-388

ISSN: 1311-8080 (printed version); ISSN: 1314-3395 (on-line version)

url: http://www.ijpam.eu

doi: http://dx.doi.org/10.12732/ijpam.v86i2.12

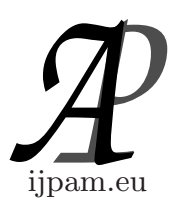

\title{
JOIN PRESERVING MAPS AND RESIDUATED CONNECTIONS
}

\author{
Yong Chan Kim \\ Department of Mathematics \\ Gangneung-Wonju National University \\ Gangneung, Gangwondo, 210-702, KOREA
}

\begin{abstract}
In this paper, we investigate the relations between join preserving (meet preserving ,join-meet, meet-join) maps and residuated (dual residuated, residuated, Galois, dual Galois) connections in complete residuated lattices.
\end{abstract}

AMS Subject Classification: 03E72, 03G10, 06A15, 06F07

Key Words: complete residuated lattices, join preserving (meet preserving, join-meet, meet-join) maps, residuated (dual residuated, residuated, Galois, dual Galois) connections

\section{Introduction}

Hájek [4] introduced a complete residuated lattice which is an algebraic structure for many valued logic. Bělohlávek [1], [2] developed the notion of fuzzy contexts using Galois connections with $R \in L^{X \times Y}$ on a complete residuated lattice. Zhang [9], [10] introduced the fuzzy complete lattice which is defined by join and meet on fuzzy posets. It is an important mathematical tool for algebraic structure of fuzzy contexts [1]-[3], [5]-[8].

In this paper, we investigate the relations between join preserving (meet preserving, join-meet, meet-join) maps and residuated (dual residuated, residuated, Galois, dual Galois) connections in complete residuated lattices.

Received: February 22, 2013

(c) 2013 Academic Publications, Ltd. url: www.acadpubl.eu 
Definition 1.1. (see [1], [4]) An algebra $(L, \wedge, \vee, \odot, \rightarrow, 0,1)$ is called a complete residuated lattice if it satisfies the following conditions:

(C1) $L=(L, \leq, \vee, \wedge, 1,0)$ is a complete lattice with the greatest element 1 and the least element 0 ;

(C2) $(L, \odot, 1)$ is a commutative monoid;

(C3) $x \odot y \leq z$ iff $x \leq y \rightarrow z$ for $x, y, z \in L$.

In this paper, we assume $\left(L, \wedge, \vee, \odot, \rightarrow,{ }^{*} 0,1\right)$ is a complete residuated lattice with the law of double negation;i.e. $x^{* *}=x$.

Lemma 1.2. (see [1], [4]) For each $x, y, z, x_{i}, y_{i} \in L$, we have the following properties.

(1) If $y \leq z,(x \odot y) \leq(x \odot z), x \rightarrow y \leq x \rightarrow z$ and $z \rightarrow x \leq y \rightarrow x$.

(2) $x \rightarrow\left(\bigwedge_{i \in \Gamma} y_{i}\right)=\bigwedge_{i \in \Gamma}\left(x \rightarrow y_{i}\right)$.

(3) $\left(\bigvee_{i \in \Gamma} x_{i}\right) \rightarrow y=\bigwedge_{i \in \Gamma}\left(x_{i} \rightarrow y\right)$.

(4) $\bigwedge_{i \in \Gamma} y_{i}^{*}=\left(\bigvee_{i \in \Gamma} y_{i}\right)^{*}$ and $\bigvee_{i \in \Gamma} y_{i}^{*}=\left(\bigwedge_{i \in \Gamma} y_{i}\right)^{*}$.

(5) $(x \odot y) \rightarrow z=x \rightarrow(y \rightarrow z)=y \rightarrow(x \rightarrow z)$.

(6) $x \odot y=\left(x \rightarrow y^{*}\right)^{*}$ and $x \rightarrow y=y^{*} \rightarrow x^{*}$.

(7) $x \odot(x \rightarrow y) \leq y$.

(8) $(x \rightarrow y) \odot(y \rightarrow z) \leq x \rightarrow z$.

(9) $x \leq y \rightarrow z$ iff $y \leq x \rightarrow z$.

Definition 1.3. (see [9], [10]) Let $X$ be a set. A function $e_{X}: X \times X \rightarrow L$ is called:

(E1) reflexive if $e_{X}(x, x)=1$ for all $x \in X$,

(E2) transitive if $e_{X}(x, y) \odot e_{X}(y, z) \leq e_{X}(x, z)$, for all $x, y, z \in X$,

(E3) if $e_{X}(x, y)=e_{X}(y, x)=1$, then $x=y$.

If $e$ satisfies (E1) and (E2), $\left(X, e_{X}\right)$ is a fuzzy preorder set. If $e$ satisfies (E1), (E2) and (E3), $\left(X, e_{X}\right)$ is a fuzzy partially order set (simply, fuzzy poset).

Remark 1.4. (1) We define a function $e_{L^{X}}: L^{X} \times L^{X} \rightarrow L$ as $e_{L^{X}}(A, B)=$ $\bigwedge_{x \in X}(A(x) \rightarrow B(x))$. Then $\left(L^{X}, e_{L^{X}}\right)$ is a fuzzy poset from Lemma $1.2(8)$. 
Definition 1.5. (see [1], [3]) Let $\left(X, e_{X}\right)$ and $\left(Y, e_{Y}\right)$ be a fuzzy poset and $f: X \rightarrow Y$ and $g: Y \rightarrow X$ maps.

(1) $\left(e_{X}, f, g, e_{Y}\right)$ is called a Galois connection if for all $x \in X, y \in Y$,

$$
e_{Y}(y, f(x))=e_{X}(x, g(y)) .
$$

(2) $\left(e_{X}, f, g, e_{Y}\right)$ is called a dual Galois connection if for all $x \in X, y \in Y$,

$$
e_{Y}(f(x), y)=e_{X}(g(y), x) .
$$

(3) $\left(e_{X}, f, g, e_{Y}\right)$ is called a residuated connection if for all $x \in X, y \in Y$,

$$
e_{Y}(f(x), y)=e_{X}(x, g(y)) .
$$

(4) $\left(e_{X}, f, g, e_{Y}\right)$ is called a dual residuated connection if for all $x \in X, y \in$ $Y$,

$$
e_{Y}(y, f(x))=e_{X}(g(y), x) .
$$
$L^{X}$.

Definition 1.6. (see [5], [9], [10]) Let $\left(X, e_{X}\right)$ be a fuzzy poset and $A \in$

A point $x_{0}$ is called a join of $A$, denoted by $x_{0}=\sqcup A$, if it satisfies

(J1) $A(x) \leq e_{X}\left(x, x_{0}\right)$,

(J2) $\bigwedge_{x \in X}\left(A(x) \rightarrow e_{X}(x, y)\right) \leq e_{X}\left(x_{0}, y\right)$.

A point $x_{1}$ is called a meet of $A$, denoted by $x_{1}=\sqcap A$, if it satisfies

(M1) $A(x) \leq e_{X}\left(x_{1}, x\right)$,

(M2) $\bigwedge_{x \in X}\left(A(x) \rightarrow e_{X}(y, x)\right) \leq e_{X}\left(y, x_{1}\right)$.

The pair $\left(X, e_{X}\right)$ is called a fuzzy complete lattice if for all $A \in L^{X}, \sqcup A$ and $\sqcap A$ exist.

Let $\left(L^{X}, e_{L^{X}}\right)$ and $\left(L^{Y}, e_{L^{Y}}\right)$ be a fuzzy poset and $\eta: L^{X} \rightarrow L^{Y}$ a map. We define $\eta^{\rightarrow}: L^{L^{X}} \rightarrow L^{L^{Y}}, \eta^{\leftarrow}: L^{L^{Y}} \rightarrow L^{L^{X}}$ as

$$
\eta \rightarrow(\mathcal{U})(B)=\bigvee_{\eta(A)=B} \mathcal{U}(A), \quad \eta^{\leftarrow}(\mathcal{V})(A)=\mathcal{V}(\eta(A))
$$

(1) $\eta$ is a join preserving map if $\eta(\sqcup \mathcal{U})=\sqcup \eta \rightarrow(\mathcal{U})$ for all $\mathcal{U} \in L^{L^{X}}$.

(2) $\eta$ is a meet preserving map if $\eta(\sqcap \mathcal{U})=\sqcap \eta \rightarrow(\mathcal{U})$ for all $\mathcal{U} \in L^{L^{X}}$.

(3) $\eta$ is a meet-join map if $\eta(\sqcap \mathcal{U})=\sqcup \eta \rightarrow(\mathcal{U})$ for all $\mathcal{U} \in L^{L^{X}}$. 
(4) $\eta$ is a join-meet map if $\eta(\sqcup \mathcal{U})=\sqcap \eta \rightarrow(\mathcal{U})$ for all $\mathcal{U} \in L^{L^{X}}$.

In this paper, $1_{x}: X \rightarrow Y$ is a characteristic function,i.e. $1_{x}(x)=1$, otherwise $1_{x}(y)=0$. Moreover $\uparrow A, \downarrow B: L^{X} \rightarrow L^{X}$ is defined as $\uparrow A(C)=$ $e_{L^{X}}(A, C), \downarrow B(C)=e_{L^{X}}(C, B)$.

Remark 1.7. (see [5]) Let $\left(L, e_{L}\right)$ be a fuzzy poset and $A \in L^{L}$.

(1) Since $x_{0}$ is a join of $A$ iff $\bigwedge_{x \in L}\left(A(x) \rightarrow e_{L}(x, y)\right)=\bigwedge_{x \in L}(A(x) \rightarrow$ $(x \Rightarrow y))=\bigvee_{x \in L}(x \odot A(x)) \rightarrow y=e_{L}\left(x_{0}, y\right)=x_{0} \rightarrow y$, then $x_{0}=\sqcup A=$ $\bigvee_{x \in L}(x \odot A(x))$.

(2) Since $x_{0}$ is a join of $A$ iff $\bigwedge_{x \in L}\left(A(x) \rightarrow e_{L}(x, y)\right)=\bigwedge_{x \in L}(A(x) \rightarrow(y \rightarrow$ $x))=\bigwedge_{x \in L}(y \rightarrow(A(x) \rightarrow x))=y \rightarrow \bigwedge_{x \in L}(A(x) \rightarrow x)=y \rightarrow \sqcap A$, then $\sqcap A=\bigwedge_{x \in L}(A(x) \rightarrow x)$.

Remark 1.8. (see [5]) Let $\left(L^{X}, e_{L^{X}}\right)$ be a fuzzy poset and $\mathcal{U} \in L^{L^{X}}$.

(1) Since $\bigwedge_{A \in L^{X}}\left(\mathcal{U}(A) \rightarrow e_{L^{X}}(A, B)\right)=e_{L^{X}}\left(\bigvee_{A \in L^{X}}(\mathcal{U}(A) \odot A), B\right)=$ $e_{L^{X}}(\sqcup \mathcal{U}, B)$, then $\sqcup \mathcal{U}=\bigvee_{A \in L^{X}}(\mathcal{U}(A) \odot A)$.

(2) Since $\bigwedge_{A \in L^{X}}\left(\mathcal{U}(A) \rightarrow e_{L^{X}}(B, A)=\bigwedge_{A \in L^{X}} e_{L^{X}}(B,(\mathcal{U}(A) \rightarrow A))=\right.$ $e_{L^{X}}\left(B, \bigwedge_{A \in L^{X}}(\mathcal{U}(A) \rightarrow A)\right)$, then $\sqcap \mathcal{U}=\bigwedge_{A \in L^{X}}(\mathcal{U}(A) \rightarrow A)$.

Theorem 1.9. (see [5]) Let $\left(L^{X}, e_{L^{X}}\right)$ and $\left(L^{Y}, e_{L^{Y}}\right)$ be fuzzy posets and $\mathcal{U} \in L^{L^{X}}$. Then the following statements hold.

(1) $\eta\left(\bigvee_{i \in \Gamma} A_{i}\right)=\bigwedge_{i \in \Gamma} \eta\left(A_{i}\right)$ and $\eta(\alpha \odot A)=\alpha \rightarrow \eta(A)$ iff $\eta(\sqcup \mathcal{U})=\sqcap \eta \rightarrow(\mathcal{U})$ for all $\mathcal{U} \in L^{L^{X}}$.

(2) $\phi\left(\bigvee_{i \in \Gamma^{X}} A_{i}\right)=\bigvee_{i \in \Gamma} \phi\left(A_{i}\right)$ and $\phi(\alpha \odot A)=\alpha \odot \phi(A)$ iff $\phi(\sqcup \mathcal{U})=\sqcup \phi \rightarrow(\mathcal{U})$ for all $\mathcal{U} \in L^{L^{X}}$.

(3) $\omega\left(\bigwedge_{i \in \Gamma} A_{i}\right)=\bigvee_{i \in \Gamma} \omega\left(A_{i}\right)$ and $\omega(\alpha \rightarrow A)=\alpha \odot \omega(A)$ iff $\omega(\sqcap \mathcal{U})=$ $\sqcup \omega \rightarrow(\mathcal{U})$ for all $\mathcal{U} \in L^{L^{X}}$.

(4) $\psi\left(\bigwedge_{i \in \Gamma} A_{i}\right)=\bigwedge_{i \in \Gamma} \psi\left(A_{i}\right)$ and $\psi(\alpha \rightarrow A)=\alpha \rightarrow \psi(A)$ iff $\psi(\sqcap \mathcal{U})=$ $\sqcap \psi \rightarrow(\mathcal{U})$ for all $\mathcal{U} \in L^{L^{X}}$.

\section{Join Preserving Maps and Residuated Connections}

Theorem 2.1. Let $\left(L^{X}, e_{L^{X}}\right)$ and $\left(L^{Y}, e_{L^{Y}}\right)$ be fuzzy posets and $\mathcal{U} \in L^{L^{X}}$. Then the following statements hold. 
(1) $\eta(\sqcup \mathcal{U})=\sqcap \eta \rightarrow(\mathcal{U})$ for all $\mathcal{U} \in L^{L^{X}}$ iff there exists $\theta: L^{Y} \rightarrow L^{X}$ with

$$
\begin{aligned}
\theta(B) & =\bigvee\left\{A \in L^{X} \mid B \leq \eta(A)\right\}=\sqcup \eta^{\leftarrow}(\uparrow B) \\
& =\bigvee_{C \in L^{X}}\left(e_{L^{Y}}(B, \eta(C)) \odot C\right)
\end{aligned}
$$

such that $\left(e_{L^{X}}, \eta, \theta, e_{L^{Y}}\right)$ is a Galois connection.

(2) $\phi(\sqcup \mathcal{U})=\sqcup \phi \rightarrow(\mathcal{U})$ for all $\mathcal{U} \in L^{L^{X}}$ iff there exists $\gamma: L^{Y} \rightarrow L^{X}$ with

$$
\begin{aligned}
\gamma(B) & =\bigvee\left\{A \in L^{X} \mid \phi(A) \leq B\right\}=\sqcup \phi^{\leftarrow}(\downarrow B) \\
& =\bigvee_{C \in L^{X}}\left(e_{L^{Y}}(\phi(C), B) \odot C\right)
\end{aligned}
$$

such that $\left(e_{L^{X}}, \phi, \gamma, e_{L^{Y}}\right)$ is a residuated connection.

(3) $\omega(\sqcap \mathcal{U})=\sqcup \omega \rightarrow(\mathcal{U})$ for all $\mathcal{U} \in L^{L^{X}}$ iff there exists $\rho: L^{Y} \rightarrow L^{X}$ with

$$
\begin{aligned}
& \rho(B)=\bigwedge\left\{A \in L^{X} \mid \omega(A) \leq B\right\}=\sqcap \omega^{\leftarrow}(\downarrow B) \\
& =\bigwedge_{C \in L^{X}}\left(e_{L^{Y}}(\omega(C), B) \rightarrow C\right)
\end{aligned}
$$

such that $\left(e_{L^{X}}, \omega, \rho, e_{L^{Y}}\right)$ is an dual Galois connection. with

(4) If $\psi(\sqcap \mathcal{U})=\sqcap \psi \rightarrow(\mathcal{U})$ for all $\mathcal{U} \in L^{L^{X}}$, then there exists $\tau: L^{Y} \rightarrow L^{X}$

$$
\tau(B)=\sqcap \psi^{\leftarrow}(\uparrow B)=\bigwedge_{C \in L^{X}}\left(e_{L^{Y}}(B, \psi(C)) \rightarrow C\right)=\bigwedge\left\{A \in L^{X} \mid \psi(A) \geq B\right\}
$$

such that $\left(e_{L^{X}}, \psi, \tau, e_{L^{Y}}\right)$ is a dual residuated connection.

Proof. (1) $(\Rightarrow)$ Define $\theta: L^{Y} \rightarrow L^{X}$ as $\theta(B)=\bigvee\left\{C \in L^{X} \mid B \leq \eta(C)\right\}$. By Theorem $1.9(1)$, since $\eta\left(\bigvee_{i \in \Gamma} A_{i}\right)=\bigwedge_{i \in \Gamma} \eta\left(A_{i}\right)$ and $\eta(\alpha \odot A)=\alpha \rightarrow \eta(A)$, for $C=\bigvee_{x \in X}\left(C(x) \odot 1_{x}\right)$, we have

$$
\eta(C)(y)=\bigwedge_{x \in X}\left(C(x) \rightarrow \eta\left(1_{x}\right)(y)\right)
$$

Thus,

$$
\begin{aligned}
\theta(B)(x) & =\bigvee\{C(x) \mid \eta(C) \geq B\} \\
& =\bigvee\left\{C(x) \mid \bigwedge\left(C(x) \rightarrow \eta\left(1_{x}\right)(y)\right) \geq B(y)\right\} \\
& \left.=\bigvee\left\{C(x) \mid \bigwedge\left(B(y) \rightarrow \eta\left(1_{x}\right)(y)\right) \geq C(x)\right\} \quad \text { (by Lemma } 1.2(9)\right) \\
& =\bigwedge\left(B(y) \rightarrow \eta\left(1_{x}\right)(y)\right) .
\end{aligned}
$$




$$
\begin{aligned}
& e_{L^{Y}}(B, \eta(A)) \\
& =\bigwedge_{y \in Y}\left(B(y) \rightarrow \eta\left(\bigvee_{x \in X}\left(A(x) \odot 1_{x}\right)\right)(y)\right) \\
& =\bigwedge_{y \in Y}\left(B(y) \rightarrow \bigwedge_{x \in X}\left(A(x) \rightarrow \eta\left(1_{x}\right)(y)\right)\right) \\
& =\bigwedge_{y \in Y} \bigwedge_{x \in X}\left(B(y) \rightarrow\left(A(x) \rightarrow \eta\left(1_{x}\right)(y)\right)\right) \\
& =\bigwedge_{x \in X}\left(A(x) \rightarrow \bigwedge_{y \in Y}\left(B(y) \rightarrow \eta\left(1_{x}\right)(y)\right)\right) \quad(\text { by Lemma } 1.2(5)) \\
& =\bigwedge_{x \in X}(A(x) \rightarrow \theta(B)(x))=e_{L^{X}}(A, \theta(B)) .
\end{aligned}
$$

Then $\left(e_{L^{X}}, \eta, \theta, e_{L^{Y}}\right)$ is a Galois connection.

By the definition of $\sqcup \eta^{\leftarrow}(\uparrow B)$, we have $\sqcup \eta^{\leftarrow}(\uparrow B)=\bigvee_{C \in L^{X}}\left(e_{L^{Y}}(B, \eta(C)) \odot\right.$ C) from:

$$
\begin{aligned}
e_{L^{X}}\left(\sqcup \eta^{\leftarrow}(\uparrow B), D\right) & =\bigwedge_{C \in L^{Y}}\left(\eta^{\leftarrow}(\uparrow B)(C) \rightarrow e_{L^{X}}(C, D)\right) \\
& =\bigwedge_{C \in L^{Y}}\left(e_{L^{X}}\left(\eta^{\leftarrow}(\uparrow B)(C) \odot C, D\right)\right. \\
& =\bigwedge_{C \in L^{Y}} e_{L^{X}}\left(e_{L^{Y}}(B, \eta(C)) \odot C, D\right) \\
& =e_{L^{X}}\left(\bigvee_{C \in L^{Y}}\left(e_{L^{Y}}(B, \eta(C)) \odot C\right), D\right)
\end{aligned}
$$

Finally, we will show that $\theta(B)=\bigvee_{C \in L^{X}}\left(e_{L^{Y}}(B, \eta(C)) \odot C\right)$. Since $B \odot$ $\left(e_{L^{Y}}(B, \eta(C)) \leq \eta(C)\right.$, by Theorem $1.9(1)$,

$$
\eta\left(\bigvee_{C \in L^{X}}\left(e_{L^{Y}}(B, \eta(C)) \odot C\right)\right)=\bigwedge_{C \in L^{X}}\left(e_{L^{Y}}(B, \eta(C)) \rightarrow \eta(C) \geq B\right.
$$

By the definition of $\theta, \theta(B) \geq \bigvee_{C \in L^{X}}\left(e_{L^{Y}}(B, \eta(C)) \odot C\right)$. Since $\left(e_{L^{X}}, \eta, \theta, e_{L^{Y}}\right)$ is a Galois connection,

$$
e_{L^{Y}}(B, \eta(\theta(B)))=e_{L^{X}}(\theta(B), \theta(B))=1 .
$$

Hence, $\theta(B)=e_{L^{Y}}(B, \eta(\theta(B))) \odot \theta(B) \leq \bigvee_{C \in L^{X}}\left(e_{L^{Y}}(B, \eta(C)) \odot C\right)$. Thus $\theta(B)=\bigvee_{C \in L^{X}}\left(e_{L^{Y}}(B, \eta(C)) \odot C\right)$.

$(\Leftarrow)$ Put $B_{0}=\sqcap \eta \rightarrow(\mathcal{U})$. Then

$$
\begin{aligned}
e_{L^{Y}}\left(B, B_{0}\right) & =\bigwedge_{C \in L^{Y}}\left(\eta \rightarrow(\mathcal{U})(C) \rightarrow e_{L^{Y}}(B, C)\right) \\
& =\bigwedge_{C \in L^{Y}}\left(\left(\bigvee_{\eta(A)=C} \mathcal{U}(A) \rightarrow e_{L^{Y}}(B, \eta(A))\right)\right. \\
& =\bigwedge_{A \in L^{X}}\left(\mathcal{U}(A) \rightarrow e_{L^{Y}}(B, \eta(A))\right) \\
& =\bigwedge_{A \in L^{X}}\left(\mathcal{U}(A) \rightarrow e_{L^{X}}(A, \theta(B))\right) \\
& =e_{L^{X}}(\sqcup \mathcal{U}, \theta(B))=e_{L^{Y}}(B, \eta(\sqcup \mathcal{U})) .
\end{aligned}
$$

Hence $B_{0}=\sqcap \eta \rightarrow(\mathcal{U})=\eta(\sqcup \mathcal{U})$.

$(2)(\Rightarrow)$ Define $\gamma: L^{Y} \rightarrow L^{X}$ as $\gamma(B)=\bigvee\left\{C \in L^{X} \mid \phi(C) \leq B\right\}$. By Theorem 1.9(2), since $\phi\left(\bigvee_{i \in \Gamma} A_{i}\right)=\bigvee_{i \in \Gamma} \phi\left(A_{i}\right)$ and $\phi(\alpha \odot A)=\alpha \odot \phi(A)$, for $C=\bigvee_{x \in X}\left(C(x) \odot 1_{x}\right)$, we have

$$
\phi(C)(y)=\bigvee_{x \in X}\left(C(x) \odot \phi\left(1_{x}\right)(y)\right) .
$$


Thus

$$
\begin{aligned}
& \gamma(B)(x)=\bigvee\{C(x) \mid \phi(C) \leq B\} \\
&=\bigvee\left\{C(x) \mid \bigvee\left(C(x) \odot \phi\left(1_{x}\right)(y)\right) \leq B(y)\right\} \\
&=\bigvee\left\{C(x) \mid C(x) \leq \bigwedge_{y \in Y}\left(\phi\left(1_{x}\right)(y) \rightarrow B(y)\right)\right\} \\
&=\bigwedge_{y \in Y}\left(\phi\left(1_{x}\right)(y) \rightarrow B(y)\right) \\
& e_{L^{Y}}(\phi(A), B)=\bigwedge_{y \in Y}\left(\phi\left(\bigvee_{x \in X}\left(A(x) \odot 1_{x}\right)\right)(y) \rightarrow B(y)\right) \\
&=\bigwedge_{y \in Y}\left(\bigvee_{x \in X}\left(A(x) \odot \phi\left(1_{x}\right)(y) \rightarrow B(y)\right)\right. \\
&=\bigwedge_{y \in Y} \bigwedge_{x \in X}\left(A(x) \rightarrow\left(\phi\left(1_{x}\right)(y) \rightarrow B(y)\right)\right) \\
&=\bigwedge_{x \in X}\left(A(x) \rightarrow \bigwedge_{y \in Y}\left(\phi\left(1_{x}\right)(y) \rightarrow B(y)\right)\right) \\
&=\bigwedge_{x \in X}(A(x) \rightarrow \gamma(B)(x))=e_{L^{X}}(A, \gamma(B))
\end{aligned}
$$

C) from:

By the definition of $\sqcup \phi^{\leftarrow}(\downarrow B)$, we have $\sqcup \phi^{\leftarrow}(\downarrow B)=\bigvee_{C \in L^{X}}\left(e_{L^{Y}}(\phi(C), B) \odot\right.$

$$
\begin{aligned}
e_{L^{X}}\left(\sqcup \phi^{\leftarrow}(\downarrow B), D\right) & =\bigwedge_{C \in L^{Y}}\left(\phi^{\leftarrow}(\downarrow B)(C) \rightarrow e_{L^{X}}(C, D)\right) \\
& =\bigwedge_{C \in L^{Y}}\left(e_{L^{X}}\left(\phi^{\leftarrow}(\downarrow B)(C) \odot C, D\right)\right. \\
& =\bigwedge_{C \in L^{Y}} e_{L^{X}}\left(e_{L^{Y}}(\phi(C), B) \odot C, D\right) \\
& =e_{L^{X}}\left(\bigvee_{C \in L^{Y}}\left(e_{L^{Y}}(\phi(C), B) \odot C\right), D\right)
\end{aligned}
$$

Finally, we will show that $\gamma(B)=\bigvee_{C \in L^{X}}\left(e_{L^{Y}}(\phi(C), B) \odot C\right)$. Since

$$
\phi\left(\bigvee_{C \in L^{X}}\left(e_{L^{Y}}(B, F(C)) \odot C\right)\right)=\bigvee_{C \in L^{X}}\left(e_{L^{Y}}(\phi(C), B) \odot \phi(C) \leq B,\right.
$$

by the definition of $\gamma, \gamma(B) \geq \bigvee_{C \in L^{X}}\left(e_{L^{Y}}(\phi(C), B) \odot C\right)$. Since $\left(e_{L^{X}}, \phi, \gamma, e_{L^{Y}}\right)$ is a residuated connection, then

$$
e_{L^{Y}}(\phi(\gamma(B)), B)=e_{L^{X}}(\gamma(B), \gamma(B))=1 .
$$

Hence $\gamma(B)=e_{L^{Y}}(\phi(\gamma(B)), B) \odot \gamma(B) \leq \bigvee_{C \in L^{X}}\left(e_{L^{Y}}(\phi(C), B) \odot C\right)$. Thus, $\gamma(B) \leq \bigvee_{C \in L^{X}}\left(e_{L^{Y}}(\phi(C), B) \odot C\right)$.

$(\Leftarrow)$ Put $B_{1}=\sqcup \phi^{\rightarrow}(\mathcal{U})$. Then

$$
\begin{aligned}
e_{L^{Y}}\left(B_{1}, B\right) & =\bigwedge_{C \in L^{Y}}\left(\phi \rightarrow(\mathcal{U})(C) \rightarrow e_{L^{Y}}(C, B)\right) \\
& =\bigwedge_{C \in L^{Y}}\left(\left(\bigvee_{\phi(A)=C} \mathcal{U}(A) \rightarrow e_{L^{Y}}(\phi(A), B)\right)\right. \\
& =\bigwedge_{A \in L^{X}}\left(\mathcal{U}(A) \rightarrow e_{L^{Y}}(\phi(A), B)\right) \\
& =\bigwedge_{A \in L^{X}}\left(\mathcal{U}(A) \rightarrow e_{L^{X}}(A, \rho(B))\right) \\
& =e_{L^{X}}(\sqcup \mathcal{U}, \rho(B))=e_{L^{Y}}(\phi(\sqcup \mathcal{U}), B) .
\end{aligned}
$$

Hence $B_{1}=\phi(\sqcup \mathcal{U})=\sqcup \phi^{\rightarrow}(\mathcal{U})$. 
(3) $(\Rightarrow)$ Define $\rho: L^{Y} \rightarrow L^{X}$ as $\rho(B)=\bigwedge\left\{C \in L^{X} \mid \omega(C) \leq B\right\}$. By Theorem 1.9(3), since $\omega\left(\bigwedge_{i \in \Gamma} A_{i}\right)=\bigvee_{i \in \Gamma} \omega\left(A_{i}\right)$ and $\omega(\alpha \rightarrow A)=\alpha \odot \omega(A)$, for $C=\bigwedge_{x \in X}\left(1_{x} \rightarrow C(x)\right)$, we have $\omega\left(\bigwedge_{x \in X}\left(1_{x} \rightarrow C(x)\right)\right)=\omega\left(\bigwedge_{x \in X}\left(C^{*}(x) \rightarrow\right.\right.$ $\left.\left.1_{x}^{*}\right)\right)=\bigvee\left(C^{*}(x) \odot \omega\left(1_{x}^{*}\right)(y)\right)$. Thus

$$
\begin{aligned}
\rho(B)(x) & =\bigwedge\{C(x) \mid \omega(C) \leq B\} \\
& =\bigwedge\left\{C(x) \mid \omega\left(\bigwedge_{x \in X}\left(1_{x} \rightarrow C(x)\right)\right) \leq B\right\} \\
& =\bigwedge\left\{C(x) \mid \bigvee\left(C^{*}(x) \odot \omega\left(1_{x}^{*}\right)(y) \leq B(y)\right\}\right. \\
& =\bigwedge\left\{C(x) \mid C^{*}(x) \leq \bigwedge_{y \in Y}\left(\omega\left(1_{x}^{*}\right)(y) \rightarrow B(y)\right)\right\} \\
& =\bigvee_{y \in Y}\left(\omega\left(1_{x}^{*}\right)(y) \odot B^{*}(y)\right)(\text { by Lemma 1.2 }(6))
\end{aligned}
$$

Since $a^{*} \odot b \rightarrow c=\left(a^{*} \odot b \odot c^{*}\right)^{*}=c^{*} \odot b \rightarrow a$, we have

$$
\begin{aligned}
e_{L^{Y}}(\omega(A), B) & =\bigwedge_{y \in Y}\left(\omega\left(\bigwedge_{x \in X}\left(1_{x} \rightarrow A(x)\right)\right) \rightarrow B(y)\right) \\
& =\bigwedge_{y \in Y}\left(\bigvee_{x \in X}\left(A^{*}(x) \odot \omega\left(1_{x}^{*}\right)(y) \rightarrow B(y)\right)\right. \\
& =\bigwedge_{y \in Y} \bigwedge_{x \in X}\left(\left(B^{*}(y) \odot \omega\left(1_{x}^{*}\right)(y) \rightarrow A(x)\right)\right. \\
& =\bigwedge_{x \in X}\left(\bigvee_{y \in Y}\left(B^{*}(y) \odot \omega\left(1_{x}^{*}\right)(y) \rightarrow A(x)\right)\right. \\
& =\bigwedge_{x \in X}(\rho(B)(x) \rightarrow A(x))=e_{L^{X}}(\rho(B), A)
\end{aligned}
$$

By the definition of $\sqcap \omega^{\leftarrow}(\downarrow B)$, we have $\sqcap \omega^{\leftarrow}(\downarrow B)=\bigwedge_{C \in L^{X}}\left(e_{L^{Y}}(\omega(C), B) \rightarrow\right.$ C) from:

$$
\begin{aligned}
e_{L^{X}}\left(D, \sqcup \omega^{\leftarrow}(\downarrow B)\right) & =\bigwedge_{C \in L^{Y}}\left(\omega^{\leftarrow}(\downarrow B)(C) \rightarrow e_{L^{X}}(D, C)\right) \\
& =\bigwedge_{C \in L^{Y}}\left(e_{L^{X}}\left(\omega^{\leftarrow}(\downarrow B)(C) \odot D, C\right)\right. \\
& =\bigwedge_{C \in L^{Y}} e_{L^{X}}\left(e_{L^{Y}}(\omega(C), B) \odot D, C\right) \\
& =e_{L^{X}}\left(D, \bigwedge_{C \in L^{Y}}\left(e_{L^{Y}}(\omega(C), B) \rightarrow C\right)\right) .
\end{aligned}
$$

Finally, we will show that $\rho(B)=\bigwedge_{C \in L^{X}}\left(e_{L^{Y}}(\omega(C), B) \rightarrow C\right)$. Since

$$
\omega\left(\bigwedge_{C \in L^{X}}\left(e_{L^{Y}}(\omega(C), B) \rightarrow C\right)=\bigvee_{C \in L^{X}}\left(e_{L^{Y}}(\omega(C), B) \odot \omega(C) \leq B,\right.\right.
$$

by the definition of $\rho, \rho(B) \leq \bigwedge_{C \in L^{X}}\left(e_{L^{Y}}(\omega(C), B) \rightarrow C\right)$. Since $\left(e_{L^{X}}, \omega, \rho, e_{L^{Y}}\right)$ is an dual Galois connection,

$$
e_{L^{Y}}(\omega(\rho(B)), B)=e_{L^{X}}(\rho(B), \rho(B))=1 .
$$

Hence, $\rho(B)=e_{L^{Y}}(\omega(\rho(B)), B) \rightarrow \rho(B) \geq \bigwedge_{C \in L^{X}}\left(e_{L^{Y}}(\omega(C), B) \rightarrow C\right)$.

$(\Leftarrow)$ Put $B_{1}=\sqcup \omega \rightarrow(\mathcal{U})$. Then

$$
\begin{aligned}
e_{L^{Y}}\left(B_{1}, B\right) & =\bigwedge_{C \in L^{Y}}\left(\omega \rightarrow(\mathcal{U})(C) \rightarrow e_{L^{Y}}(C, B)\right) \\
& =\bigwedge_{C \in L^{Y}}\left(\left(\bigvee_{\omega(A)=C} \mathcal{U}(A) \rightarrow e_{L^{Y}}(\omega(A), B)\right)\right. \\
& =\bigwedge_{C \in L^{Y}} \bigwedge_{\omega(A)=C}\left(\mathcal{U}(A) \rightarrow e_{L^{Y}}(\omega(A), B)\right) \\
& =\bigwedge_{A \in L^{X}}\left(\mathcal{U}(A) \rightarrow e_{L^{X}}(\rho(B), A)\right) \\
& =e_{L^{X}}(\rho(B), \sqcap \mathcal{U})=e_{L^{Y}}(\omega(\sqcap \mathcal{U}), B) .
\end{aligned}
$$


Hence $B_{1}=\omega(\sqcap \mathcal{U})=\sqcup \omega \rightarrow(\mathcal{U})$.

(4) $(\Rightarrow)$ Define $\tau: L^{Y} \rightarrow L^{X}$ as $\tau(B)=\bigwedge\left\{C \in L^{X} \mid \psi(C) \geq B\right\}$. By Theorem 1.9(4), since $\psi\left(\bigwedge_{i \in \Gamma} A_{i}\right)=\bigwedge_{i \in \Gamma} \psi\left(A_{i}\right)$ and $\psi(\alpha \rightarrow A)=\alpha \rightarrow \psi(A)$, for $C=\bigwedge_{x \in X}\left(1_{x} \rightarrow C(x)\right)$, we have $\psi\left(\bigwedge_{x \in X}\left(1_{x} \rightarrow C(x)\right)\right)=\psi\left(\bigwedge_{x \in X}\left(C^{*}(x) \rightarrow\right.\right.$ $\left.\left.1_{x}^{*}\right)\right)=\bigwedge_{x \in X}\left(C^{*}(x) \rightarrow \psi\left(1_{x}^{*}\right)\right)$.

Since $\psi\left(\bigwedge_{x \in X}\left(1_{x} \rightarrow C(x)\right)\right)=\bigwedge_{x \in X}\left(C^{*}(x) \rightarrow \psi\left(1_{x}^{*}\right)\right)$, we have

$$
\begin{aligned}
\tau(B)(x) & =\bigwedge\{C(x) \mid \psi(C) \geq B\} \\
& =\bigwedge\left\{C(x) \mid \bigwedge_{x \in X}\left(C^{*}(x) \rightarrow \psi\left(1_{x}^{*}\right)\right) \geq B\right\} \\
& =\bigwedge\left\{C(x) \mid \bigwedge_{y \in Y}\left(B(y) \rightarrow \psi\left(1_{x}^{*}\right)\right) \geq C^{*}(x)\right\} \\
& =\bigwedge\left\{C(x) \mid \bigvee_{y \in Y}\left(B(y) \odot \psi\left(1_{x}^{*}\right)^{*}(y)\right) \leq C(x)\right\} \\
& =\bigvee_{y \in Y}\left(B(y) \odot \psi\left(1_{x}^{*}\right)^{*}(y)\right) .
\end{aligned}
$$

$$
\begin{aligned}
& e_{L^{X}}(\tau(B), A) \\
& =\bigwedge_{x \in X}(\tau(B)(x) \rightarrow A(x)) \\
& =\bigwedge_{x \in X}\left(\bigvee_{y \in Y}\left(B(y) \odot \psi\left(1_{x}^{*}\right)^{*}(y)\right) \rightarrow A(x)\right) \\
& =\bigwedge_{x \in X} \bigwedge_{y \in Y}\left(B(y) \rightarrow\left(\psi\left(1_{x}^{*}\right)^{*}(y) \rightarrow A(x)\right)\right) \\
& =\bigwedge_{y \in Y}\left(B(y) \rightarrow \bigwedge_{x \in X}\left(A^{*}(x) \rightarrow \psi\left(1_{x}^{*}\right)(y)\right)\right)(\text { by Lemma 1.2(6)) } \\
& =\bigwedge_{y \in Y}(B(y) \rightarrow \psi(A)(y))=e_{L^{X}}(B, \psi(A)) .
\end{aligned}
$$

By the definition of $\sqcap \psi^{\leftarrow}(\uparrow B)$, we have

$$
\sqcap \psi^{\leftarrow}(\uparrow B)=\bigwedge_{C \in L^{X}}\left(e_{L^{Y}}(B, \psi(C)) \rightarrow C\right)
$$

from:

$$
\begin{aligned}
e_{L^{X}}\left(D, \sqcap \psi^{\leftarrow}(\uparrow B)\right) & =\bigwedge_{C \in L^{Y}}\left(\psi^{\leftarrow}(\uparrow B)(C) \rightarrow e_{L^{X}}(D, C)\right) \\
& =\bigwedge_{C \in L^{Y}}\left(e_{L^{X}}\left(\psi^{\leftarrow}(\uparrow B)(C) \odot D, C\right)\right. \\
& =\bigwedge_{C \in L^{Y}} e_{L^{X}}\left(e_{L^{Y}}(B, \psi(C)) \odot D, C\right) \\
& =e_{L^{X}}\left(D, \bigwedge_{C \in L^{Y}}\left(e_{L^{Y}}(B, \psi(C)) \rightarrow C\right)\right) .
\end{aligned}
$$

Finally, we will show that

$$
\tau(B)=\bigwedge_{C \in L^{X}}\left(e_{L^{Y}}(B, \psi(C)) \rightarrow C\right) .
$$

Since $e_{L^{Y}}(B, \psi(C)) \odot B \leq \psi(C)$ implies $B \leq e_{L^{Y}}(B, \psi(C)) \rightarrow \psi(C)$, we have

$$
\psi\left(\bigwedge_{C \in L^{X}}\left(e_{L^{Y}}(B, \psi(C)) \rightarrow C\right)=\bigwedge_{C \in L^{X}}\left(e_{L^{Y}}(B, \psi(C)) \rightarrow \psi(C) \geq B .\right.\right.
$$


By the definition of $\tau$,

$$
\tau(B) \leq \bigwedge_{C \in L^{X}}\left(e_{L^{Y}}(\psi(C), B) \rightarrow C\right) .
$$

Since $\left(e_{L^{X}}, \psi, \tau, e_{L^{Y}}\right)$ is a dual residuated connection,

$$
e_{L^{X}}(\psi(\tau(B)), B)=e_{L^{Y}}(\tau(B), \tau(B))
$$

Hence, $\left.\tau(B)=e_{L^{Y}}(\psi(\tau(B)), B) \rightarrow \tau(B)\right) \geq \bigwedge_{C \in L^{X}}\left(e_{L^{Y}}(\psi(C), B) \rightarrow C\right)$. Thus $\tau(B)=\bigwedge_{C \in L^{X}}\left(e_{L^{Y}}(\psi(C), B) \rightarrow C\right)$.

$(\Leftarrow)$ Put $B_{0}=\sqcap \phi \rightarrow(\mathcal{U})$. Then

$$
\begin{aligned}
e_{L^{Y}}\left(B, B_{0}\right) & =\bigwedge_{C \in L^{Y}}\left(\psi \rightarrow(\mathcal{U})(C) \rightarrow e_{L^{Y}}(B, C)\right) \\
& =\bigwedge_{C \in L^{Y}}\left(\left(\bigvee_{\psi(A)=C} \mathcal{U}(A) \rightarrow e_{L^{Y}}(B, \psi(A))\right)\right. \\
& =\bigwedge_{A \in L^{X}}\left(\mathcal{U}(A) \rightarrow e_{L^{Y}}(B, \psi(A))\right) \\
& =\bigwedge_{A \in L^{X}}\left(\mathcal{U}(A) \rightarrow e_{L^{X}}(\tau(B), A)\right) \\
& =e_{L^{X}}(\tau(B), \sqcap \mathcal{U})=e_{L^{Y}}(B, \psi(\sqcap \mathcal{U}))
\end{aligned}
$$

Hence $B_{0}=\psi(\sqcap \mathcal{U})=\sqcap \psi \rightarrow(\mathcal{U})$.

Theorem 2.2. Let $R \in L^{X \times Y}$ be a fuzzy relation. Define maps $\eta_{R}, \phi_{R}$, $\omega_{R}, \psi_{R}: L^{X} \rightarrow L^{Y}$ as follows:

$$
\begin{aligned}
& \eta_{R}(A)(y)=\bigwedge_{x \in X}(A(x) \rightarrow R(x, y)), \\
& \phi_{R}(A)(y)=\bigvee_{x \in X}(A(x) \odot R(x, y)), \\
& \omega_{R}(A)(y)=\bigvee_{x \in X}\left(A^{*}(x) \odot R(x, y)\right), \\
& \psi_{R}(A)(y)=\bigwedge_{x \in X}(R(x, y) \rightarrow A(x))
\end{aligned}
$$

Then the following statements hold.

(1) There exists $\theta_{R}: L^{Y} \rightarrow L^{X}$ with $\theta_{R}(B)(x)=\bigwedge_{y \in Y}(B(y) \rightarrow R(x, y))$ such that $\left(e_{L^{X}}, \eta_{R}, \theta_{R}, e_{L^{Y}}\right)$ is a Galois connection.

(2) There exists $\gamma_{R}: L^{Y} \rightarrow L^{X}$ with $\gamma_{R}(B)(x)=\bigwedge_{y \in Y}(R(x, y) \rightarrow B(y))$ such that $\left(e_{L^{X}}, \phi_{R}, \gamma_{R}, e_{L^{Y}}\right)$ is a residuated connection.

(3) There exists $\rho_{R}: L^{Y} \rightarrow L^{X}$ with $\rho_{R}(B)(x)=\bigvee_{y \in Y}\left(R(x, y) \odot B^{*}(y)\right)$ such that $\left(e_{L^{X}}, \omega_{R}, \rho_{R}, e_{L^{Y}}\right)$ is an dual Galois connection.

(4) There exists $\tau_{R}: L^{Y} \rightarrow L^{X}$ with $\tau_{R}(B)(y)=\bigvee_{y \in Y}(B(y) \odot R(x, y))$ such that $\left(e_{L^{X}}, \psi_{R}, \tau_{R}, e_{L^{Y}}\right)$ is a dual residuated connection. 
Proof. (1) Since $\eta_{R}\left(\bigvee_{i \in \Gamma} A_{i}\right)=\bigwedge_{i \in \Gamma} \eta_{R}\left(A_{i}\right)$ and $\eta_{R}(\alpha \odot A)=\bigwedge_{x \in X}((\alpha \odot$ $A(x)) \rightarrow R(x, y))=\alpha \rightarrow \bigwedge_{x \in X}(A(x) \rightarrow R(x, y))=\alpha \rightarrow \eta_{R}(A)$, by Theorem $1.9(1)$, we have $\eta_{R}(\sqcup \mathcal{U})=\sqcap \eta_{R}(\mathcal{U})$ for all $\mathcal{U} \in L^{L^{X}}$. By Theorem 2.1 (1), there exists $\theta_{R}$ such that $\left(e_{L^{X}}, \eta_{R}, \theta_{R}, e_{L^{Y}}\right)$ is a Galois connection with

$$
\begin{aligned}
& \theta_{R}(B)(x)=\sqcup \eta_{R}^{\overleftarrow{R}}(\uparrow B)(x)=\bigvee_{C \in L^{X}}\left(e_{L^{Y}}\left(B, \eta_{R}(C)\right) \odot C(x)\right. \\
& =\bigvee\left\{A(x) \mid B \leq \eta_{R}(A)\right\}=\bigwedge_{y \in Y}\left(B(y) \rightarrow \eta_{R}\left(1_{x}\right)(y)\right) \\
& =\bigwedge_{y \in Y}(B(y) \rightarrow R(x, y)) \text {. }
\end{aligned}
$$

(2) Since $\phi_{R}\left(\bigvee_{i \in \Gamma} A_{i}\right)=\bigvee_{i \in \Gamma} \phi_{R}\left(A_{i}\right)$ and $\phi_{R}(\alpha \odot A)=\alpha \odot \phi_{R}(A)$, by Theorem 1.9(2), we have $\phi_{R}(\sqcup \mathcal{U})=\sqcup \phi_{R}(\mathcal{U})$ for all $\mathcal{U} \in L^{L^{X}}$. By Theorem $2.1(2)$, there exists $\gamma_{R}$ such that $\left(e_{L^{X}}, \phi_{R}, \gamma_{R}, e_{L^{Y}}\right)$ is a residuated connection with

$$
\begin{aligned}
\gamma_{R}(B)(x) & =\sqcup \phi_{R}^{\overleftarrow{R}}(\downarrow B)(x)=\bigvee_{C \in L^{X}}\left(e_{L^{Y}}\left(\phi_{R}(C), B\right) \odot C(x)\right) \\
& =\bigvee\left\{A(x) \mid \phi_{R}(A) \leq B\right\}=\bigwedge_{y \in Y}\left(\phi_{R}\left(1_{x}\right)(y) \rightarrow B(y)\right) \\
& =\bigwedge_{y \in Y}(R(x, y) \rightarrow B(y))
\end{aligned}
$$

(3) Since $\omega_{R}\left(\bigwedge_{i \in \Gamma} A_{i}\right)=\bigvee_{i \in \Gamma} \omega_{R}\left(A_{i}\right)$ and $\omega_{R}(\alpha \rightarrow A)=\bigvee_{x \in X}((\alpha \rightarrow$ $\left.A)^{*}(x) \odot R(x, y)\right)=\bigvee_{x \in X}\left(\left(\alpha \odot A^{*}(x) \odot R(x, y)\right)=\alpha \odot \omega_{R}(A)\right.$, by Theorem 1.9(3), $\omega_{R}(\sqcap \mathcal{U})=\sqcup \omega_{R}(\mathcal{U})$ for all $\mathcal{U} \in L^{L^{X}}$. By Theorem 2.1(3), there exists $\rho_{R}$ such that $\left(e_{L^{X}}, \omega_{R}, \rho_{R}, e_{L^{Y}}\right)$ is an dual Galois connection with

$$
\begin{aligned}
\rho(B)(x) & =\sqcap \omega_{R}^{\overleftarrow{R}}(\downarrow B)(x)=\bigwedge_{C \in L^{X}}\left(e_{L^{Y}}\left(\omega_{R}(C), B\right) \rightarrow C(x)\right) \\
& =\bigwedge\left\{A(x) \mid \omega_{R}(A) \leq B\right\}=\bigvee_{y \in Y}\left(\omega_{R}\left(1_{x}^{*}\right)(y) \odot B^{*}(y)\right) \\
& =\bigvee_{y \in Y}\left(R(x, y) \odot B^{*}(y)\right)
\end{aligned}
$$

(4) Since $\psi_{R}\left(\bigwedge_{i \in \Gamma} A_{i}\right)=\bigwedge_{i \in \Gamma} \psi_{R}\left(A_{i}\right)$ and $\psi_{R}(\alpha \rightarrow A)=\bigwedge_{x \in X}(R(x, y) \rightarrow$ $(\alpha \rightarrow A(x)))=\bigwedge_{x \in X}(\alpha \rightarrow(R(x, y) \rightarrow A(x)))=\alpha \rightarrow \psi_{R}(A)$, by Theorem $1.9(4), \psi_{R}(\sqcap \mathcal{U})=\sqcap \psi_{R}(\mathcal{U})$ for all $\mathcal{U} \in L^{L^{X}}$. By Theorem 2.1(4), there exists $\tau_{R}: L^{Y} \rightarrow L^{X}$ such that $\left(e_{L^{X}}, \psi_{R}, \tau_{R}, e_{L^{Y}}\right)$ is a dual residuated connection with

$$
\begin{aligned}
\tau_{R}(B)(x) & =\sqcap \psi_{R}^{\leftarrow}(\uparrow B)(x)=\bigwedge_{C \in L^{X}}\left(e_{L^{Y}}\left(B, \psi_{R}(C)\right) \rightarrow C(x)\right) \\
& =\bigwedge\left\{A \in L^{X} \mid \psi_{R}(A) \geq B\right\}=\bigvee_{y \in Y}\left(B(y) \odot \psi_{R}\left(1_{x}^{*}\right)^{*}(y)\right) \\
& =\bigvee_{y \in Y}(B(y) \odot R(x, y)) .
\end{aligned}
$$




\section{References}

[1] R. Bělohlávek, Fuzzy Galois connections, Math. Log. Quart., 45 (1999), 497-504.

[2] R. Bělohlávek, Concept lattices and order in fuzzy logic, Ann. Pure Appl. Logic, 128 (2004), 277-298.

[3] J.G. Garcia, I.M. Perez, M.A. Vicente, D. Zhang, Fuzzy Galois connections categorically, Math. Log. Quart., 56 (2010), 131-147.

[4] P. Hájek, Metamathematices of Fuzzy Logic, Kluwer Academic Publishers, Dordrecht (1998).

[5] Y.C. Kim, J.M. Ko, Maps on fuzzy comlete lattices, Submited to: Int. J. Comtemp. Math. Sciences.

[6] H. Lai, D. Zhang, Complete and directed complete $\Omega$-categories, Theoretical Computer Science, 388 (2007), 1-25.

[7] H. Lai, D. Zhang, Concept lattices of fuzzy contexts: Formal concept analysis vs. rough set theory, Int. J. Approx. Reasoning, 50 (2009), 695-707.

[8] W. Yao, L.X. Lu, Fuzzy Galois connections on fuzzy posets, Math. Log. Quart., 55(2009), 105-112.

[9] Q.Y. Zhang, L. Fan, Continuity in quantitive domains, Fuzzy Sets and Systems, 154 (2005), 118-131.

[10] Q.Y. Zhang, W.X. Xie, Fuzzy complete lattices, Fuzzy Sets and Systems, 160 (2009), 2275-2291. 